\title{
CONGENITAL ATRESIA OF POSTERIOR CHOANAE
}

\author{
BY \\ D. WALKER ASHCROFT, F.R.C.S., and A. P. NORMAN, M.B., M.R.C.P. \\ Westminster Children's Hospital
}

Congenital atresia of posterior choanae is a moderately rare condition, and although Boyd (1945) has accounted for 230 cases in the literature, description of the symptoms presenting at birth are very scanty. Unilateral choanal obstruction is very much more common than the complete bilateral obstruction that was found in the present case. Richardson (1913) described a similar case in which relays of assistants maintained an airway by keeping the mouth of the child open, until at the end of a fortnight oral breathing was established.

\section{Case Notes}

A full-term male child was noticed at birth to have a fluttery type of breathing and was immediately laryngoscoped; mucus was removed from the larynx, which itself appeared quite normal. The respirations did not become regular and when the child was seen a few hours later the mouth was open and there were periods of apnoea followed by a few very rapid gasping breaths; the breathing appeared to be almost entirely abdominal, the diaphragm seeming to go into spasm and then to cause the fluttering movements of the abdomen. There was no obvious intercostal recession, the chest remaining inflated; on auscultation the air entry was poor, but there were no added sounds.

A radiograph showed no abnormality in lungs or heart.

At first it was thought that the condition was due to partial bronchial obstruction by mucus, but the child failed to improve, and had great difficulty in taking feeds, failing entirely from the breast and almost entirely from the bottle. On the third day of life attempts were unsuccessfully made to pass first a soft rubber and then a silver Eustachian catheter through the nose. A diagnosis of bilateral imperforate posterior choanae was made; about this time a thick mucoid nasal discharge appeared and rapidly became more profuse.

On the fifth day of life it was decided that a surgical attempt sbould be made to establish a nasal airway. $A$ few drops of 1 per cent. amethocaine solution were instilled into each nostril and allowed to remain for ten minutes, with the baby lying on its back. The mouth was so small that digital examination of the nasopharynx was impossible, but exploration with a bent probe revealed the existence of a postnasal space. Using an antrum burr the posterior choanal obstruction was broken down first on one side and then on the other. The choanal partitions were much thicker than was expected and considerable force had to be used in order to penetrate them with the burr. Care was taken not to penetrate the soft palate-a mishap which can easily occur with such a tiny patient-by placing a palpating finger against the buccal surface of the palate in order to determine the position of the advancing burr head. The smallness of the nostrils precluded the use of any kind of punch forceps, and it was not possible to remove with precision any part of the posterior margin of the nasal septum. Nevertheless posterior choanal openings sufficiently large to admit the passage of a full-sized frontal sinus bougie were established.

The operation was accompanied by surprisingly little shock, and the child was brought out of the theatre breathing normally, with the mouth closed. After a few hours the mucoid nasal discharge caused a recurrence of the symptoms and it became necessary frequently to suck the nose clear. The child had to be spoon-fed, until eventually the discharge decreased and it became possible to feed him from the breast.

After operation he was treated with nasal drops, at first alkaline, and then ephedrine 0.5 per cent. in normal saline; frontal sinus bougies were passed, at first daily, and then at longer intervals. A peculiar feature of this period was persistence of a very rapid respiratory rate and the occurrence of ' grey ' turns during feeding.

He was eventually discharged one month after birth, in good condition and weighing $8 \mathrm{lb}$., his birth weight having been $7 \frac{1}{2} \mathrm{lb}$.

He was readmitted fourteen days later with an acute upper respiratory infection and nasal obstruction due to mucopurulent discharge, and he died in forty-eight hours. Post-mortem examination showed that a reasonable airway had been obtained through the posterior choanae and that the postnasal space was not constricted, although blocked by thick pus. The heart was moderately enlarged, the ductus arteriosus was widely patent, and there was an abrupt narrowing of the aorta between the left subclavian artery and the ductus, causing a 
coarctation of the foetal type. The remaining the symptoms reappeared for a while. Death from findings did not contribute to the condition.

\section{Summary}

An account is given of a case of complete bilateral posterior choanal obstruction in an infant, dramatically but temporarily relieved by operation; owing to the occurrence of a mucoid nasal discharge an upper respiratory infection occurred at the age of six weeks.

\section{RERERENCES}

Boyd, H. M. E. (1945). Arch. Otolaryng., Chicago, 41, 261.

Richardson, C. W. (1913). Trans. Amer. laryng. Ass., 35, 226. 